\title{
Computed fiber evaluation of SEM images using DiameterJ
}

\section{Capabilities and limitations}

\section{Abstract:}

Fiber materials offer a high potential for improving the surface characteristics of medical implants. For quality assurance of nano- and microfiber structures the morphology is inspected by Scanning Electron Microscopy (SEM) as a standard method. Vast quantities of image data have to be evaluated. Usual practice for obtaining the fiber diameters is the manually setting of measurement points. The software DiameterJ which runs as plugin in ImageJ automatically computes fiber diameters. Here we investigated its capabilities and limitations by comparing the evaluation of selected sample SEM images of electrospun fibers. In this study the fibers of three examplary images specified by different contrast and fiber morphology were analyzed by using varied segmentation algorithms. The results are displayed in bar charts of frequency distribution. Additionally the computed fiber diameters were compared to manual measurements. Depending on various image properties the segmentation process works more or less reliable, and fault data of incomplete segmented fibers are computed. Often the results are eligible, but frequently DiameterJ generates data resembling to thin fibers, which are not present in the image. In some cases the peaks of fault data are much higher than peaks of real fibers. In consequence misinterpretation of data cannot be avoided. DiameterJ is a validated tool with the ability to generate reliable results. Future work on improving the segmentation algorithms can refine computed evaluation.

Keywords: automated, calculation, fiber diameter, ImageJ, nanofiber, microfiber, electrospinning

\footnotetext{
*Corresponding author: Andreas Götz: Institute for Biomedical Engineering, Rostock University Medical Center, FriedrichBarnewitz-Str. 4, 18119 Rostock, Germany, andreas.goetz@unirostock.de

Volkmar Senz, Sabine IIIner, Niels Grabow: Institute for Biomedical Engineering, University Medical Center Rostock, Rostock, Germany
}

https://doi.org/10.1515/cdbme-2020-3113

\section{Introduction}

Electrospinning is a widely established method of producing micro- to nanofibers. The statistical distribution of fiber diameters comprises information about process stability and the effect of influencing factors. Scanning Electron Microscopy is the standard technique for monitoring the fiber morphology of nonwoven structures. State of the art for the evaluation of fiber diameters is the manual determination of an adequate number of measurement points in the image [1, 2]. That practice reveals two shortcomings. The first one is the personal influence: an operator selects measuring points based on feeling and experience. Thus, different persons may determine different fiber diameters. The second shortcoming of that time consuming method is the limited number of points. For reliable statistics high quantities of values are necessary.

A promising alternative are computerized evaluation procedures, which automatically generate plentiful data. DiameterJ as plugin for the extensively used software ImageJ is such a tool [3]. It is specially designed for measuring fibers in SEM images and was validated on special test images as well as SEM images of steel wires and PLGA fibers [4]. That software tool uses different algorithms and gray scale values to binarize the fiber structure and consequently separates fibers from background. The user has to choose one of different results of segmentation provided by the software. In further processing the fiber, structures are skeletonized and the fiber radii, pores, angles and other data are calculated. To study the capabilities and limitations of DiameterJ for fiber evaluation we investigated numerous images. Three examples are shown here: one image with reliable results compared to two images which reveal the limits of automated computation. 


\section{Material and Methods}

Three groups of representative SEM images of clearly visible electrospun fibers and corresponding segmented images were evaluated: A: gray fibers and dark background, B: gray fibers on gray background, and $\mathrm{C}$ : gray fibers of different thicknesses on dark background. All image processing was done using ImageJ version 1.52a, the computed evaluation was done using the plugin DiameterJ version 1-018. Three different segmentation algorithms were performed on each image to compare the results: M (Mixed), S (Stat. Region Merged), and $\mathrm{T}$ (Traditional). DiameterJ generates eight different separation images of each algorithm. The operator has to choose "the best one" for further computation. For a trusty comparableness segmented images of the same order of each algorithm were chosen, image A: M3, S3, T3, image B: M1, S1, T1, image C: M7, S7, T7. As reference, 50 manually set measurement points on each image were statistically processed.

\section{Results}

The results of the evaluation of three SEM images are displayed in Figure 1. In the left row images and frequency distribution of manually chosen fiber diameters are shown as red colored bar charts. The segmented images using the algorithms $\mathrm{M}, \mathrm{S}$, and $\mathrm{T}$ as well as the corresponding frequency distributions are shown in the other rows as blue colored bar charts. Average fiber diameters in image A were found as $0.81 \pm 0.23 \mu \mathrm{m}$ for manually measurement and 0.79 $\pm 0.25 \mu \mathrm{m}, 0.77 \pm 0.26 \mu \mathrm{m}$ and $0.69 \pm 0.35 \mu \mathrm{m}$ for algorithms $\mathrm{M}, \mathrm{S}$ and $\mathrm{T}$, respectively. In image B the manually measured fiber diameters are $1.55 \pm 0.14 \mu \mathrm{m}$, whereas in image $\mathrm{C}$ the thin fibers are about $0.2 \mu \mathrm{m}$ and the thick fibers are in a range from 1.5 to $3.5 \mu \mathrm{m}$. A remarkable difference between manual counts (50 in total) and automatically generated counts (hundreds or thousands) is clearly visible.

\section{Discussion}

We compared three segmentation algorithms in ImageJ for three characteristic SEM images of electrospun fibers. No doubt, DiameterJ is a supportive and validated tool for the evaluation of SEM images and generates beneficial data. Because of the circumstance that in several SEM images fibers are not mapped faultlessly, the segmentation process does not work correctly. In consequence fault data are generated which appear as fibers with small diameters. It is not possible for the user to distinguish between fault data and real small fibers. We see the manual measurement as reliable reference for data comparison. For image A the segmentation algorithms $\mathrm{M}$ and $\mathrm{S}$ generated a result which fits good to the manually measured values, whereas algorithm $\mathrm{T}$ generated some thin white structures in the segmentation image resulting as a half of maximum sized peak at around $0.25 \mu \mathrm{m}$ fiber diameter. Image $\mathrm{B}$ clearly reveals the limits of DiameterJ. Algorithm $M$ detected half of the fibers incompletely resulting in a peak for thin fibers. That peak of fault data is $28 \%$ higher than the peak of the actual fiber diameter. The algorithms $\mathrm{S}$ and $\mathrm{T}$ show inferior results. Due to a high fraction of faulty segmented fibers the fault peaks are 7 times and 18 times higher than those of actual fibers, respectively. The clearest difference between manually and automated evaluation reveals image C. Only thin fibers $(<1$ $\mu \mathrm{m})$ are detected by DiameterJ. Existing thick fibers (1.5-3.5 $\mu \mathrm{m})$ remained undetected because they were not segmented in full diameter. Remarkably the shape of the peaks of thin fibers and fault data seem to resemble each other: high-angle steepness at the left flank and a less inclined steepness towards increasing diameters, hence it is impossible to discriminate between real thin fibers and fault data. In most cases automated evaluations of daily routine images generate peaks with different magnitudes of nonexistent fiber diameters. Thus, the rate of false positive results is relatively high. In case there are no thin fibers in the image, the corresponding peaks can be ignored. For evaluation of numerous images DiameterJ then is advantageous. In Table 1 the main characteristics of both methods, manual and automatical evaluation, are compared.

Table 1: Evaluation methods, comparison of core characteristics

\begin{tabular}{lll}
\hline Methods & Advantages & Disadvantages \\
\hline manually measured & $\begin{array}{l}\text { exact and easy, } \\
\text { standard software }\end{array}$ & $\begin{array}{l}\text { subjective, } \\
\text { limited number of } \\
\text { measuring points }\end{array}$ \\
$\begin{array}{l}\text { automatically } \\
\text { computed using }\end{array}$ & $\begin{array}{l}\text { high amount of data } \\
\text { fully computable, } \\
\text { DiameterJ }\end{array}$ & $\begin{array}{l}\text { fault data, } \\
\text { misinterpretation }\end{array}$ \\
\hline
\end{tabular}

As the separation of thin fibers located next to thicker ones is not executed properly, the use of DiameterJ is not recommended in those cases. If only thin fibers are present in the image, the separated image has to be reviewed thoroughly, including a check for plausibility of the final results. 
SEM Images
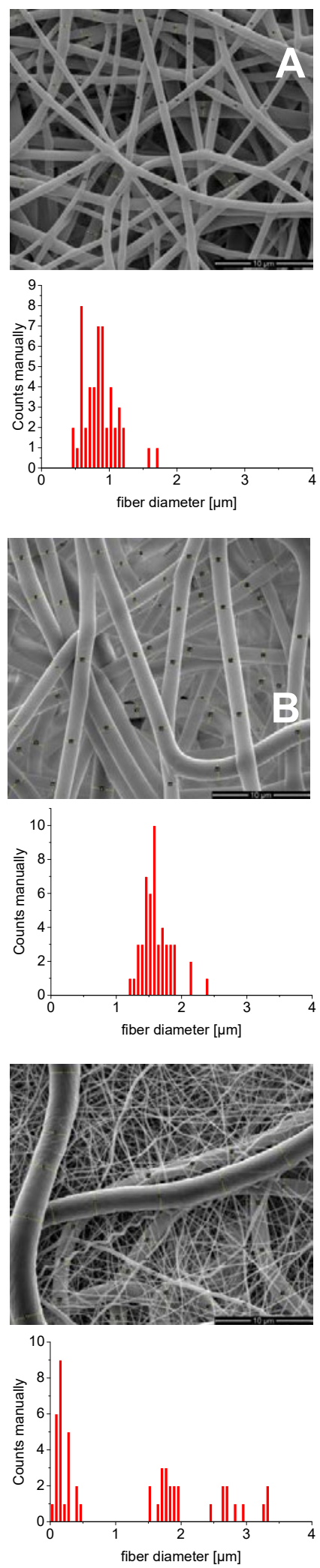

Algorithm M

Algorithm S

Algorithm T
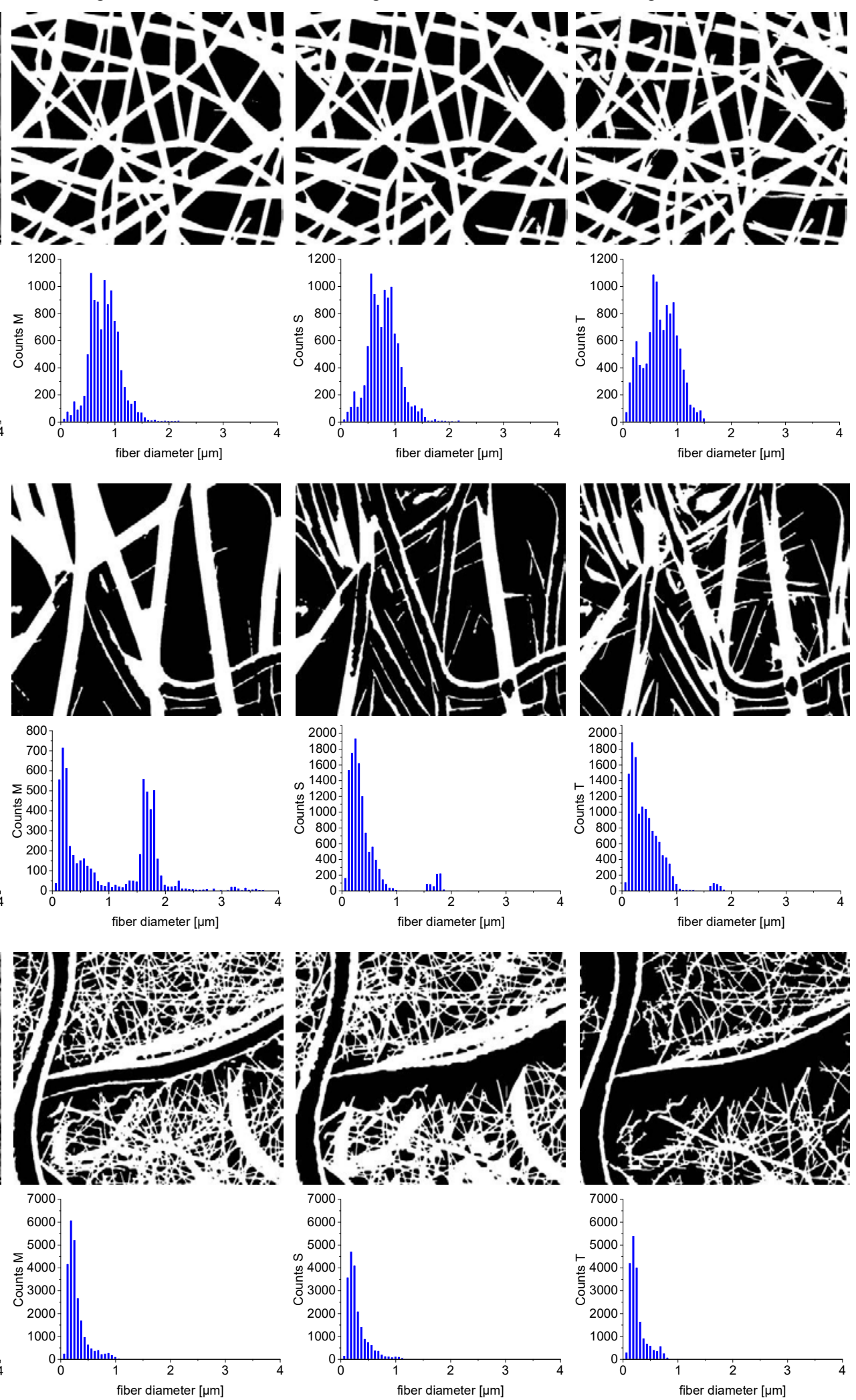
Figure 1: SEM images of electrospun fibers, scale bar $10 \mu \mathrm{m}$; Images computed by DiameterJ performing the segmentation algorithms Mixed (M), Stat. Region Merged (S), and Traditional (T); The images represent specific properties - A: good evaluable image for reliable results, B: image with less background contrast but good visible fibers, C: image with highly different fibers on dark background. In rows images and corresponding bar charts of diameter distribution are displayed. The SEM image row shows the SEM images with 50 manually set measurement points. The algorithm rows show the computed results of segmented images using algorithm $\mathrm{M}, \mathrm{S}$ and $\mathrm{T}$, respectively.

\section{Summary and Outlook}

We compared the manual evaluation method and the software tool DiameterJ to obtain diameters of electrospun fibers in SEM images. Using three exemplary images we showed the capability and limitations of DiameterJ. No segmentation algorithm revealed as the "best case". Due to faulty segmentation, bar charts of fiber diameter distribution showed peaks of nonexistent fibers at one hand and nondetected fibers at the other hand. A good image contrast is no surety for reliable results, the fiber surface seems to strongly influence the results. Performing DiameterJ on numerous SEM images of daily routine revealed the necessity for improving automated software tools.

Certainly, no software can chose measurement points with a similar intention like an individual person, fault data are unavoidable. The main advantage of automated evaluation is the processing of vast amounts of image data less influenced by the user. To improve the correctness of automatically generated data the focus is to set on better performing segmentation algorithms. Further effort can be directed at thresholds of gray scale values or on fiber surface. However, refining the segmentation algorithms is a promising approach for future works.

\section{Acknowledgment}

The authors would like to thank Jonathan Ortelt, Manfred Strotmeier, Babette Hummel and Katja Hahn for their skillful work.

\section{Author Statement}

Research funding: Partial financial support by the Federal Ministry of Education and Research (BMBF) within RESPONSE "Partnership for Innovation in Implant Technology" and by the European Social Fund (ESF) within the excellence research program of the state MecklenburgVorpommern Card-ii-Omics is gratefully acknowledged. Conflict of interest: Authors state no conflict of interest. Informed consent: Informed consent is not applicable. Ethical approval: The conducted research is not related to either human or animal use.

\section{References}

[1] Wang, Bo; Cai, Qing; Zhang, Shen; Yang, Xiaoping; Deng, Xuliang: The effect of poly (L-lactic acid) nanofiber orientation on osteogenic responses of human osteoblastlike MG63 cells, Bd. 4, S. 600-609.

[2] Zhang, Kuihua; Zheng, Honghao; Liang, Su; Gao, Changyou: Aligned PLLA nanofibrous scaffolds coated with graphene oxide for promoting neural cell growth, Bd. 37, S. 131-142.

[3] Hotaling, Nathan A.; Bharti, Kapil; Kriel, Haydn; Simon, Carl G. (2015): DiameterJ: A validated open source nanofiber diameter measurement tool. In: Biomaterials 61, S. 327-338. DOI: 10.1016/j.biomaterials.2015.05.015.

[4] Hotaling, Nathan A.; Bharti, Kapil; Kriel, Haydn; Simon, Carl G. (2015): Dataset for the validation and use of DiameterJ an open source nanofiber diameter measurement tool. In: Biomaterials 61, S. 327-338. DOI: 10.1016/j.biomaterials.2015.05.015. 